\title{
Does the Wilson frame assist with optimizing surgical exposure for minimally invasive lumbar fusions?
}

\author{
Mario J. Cardoso, M.D., D.C., and Michael K. Rosner, M.D. \\ Neurosurgery Service, Walter Reed Army Medical Center, Washington, DC
}

\begin{abstract}
Object. Minimally invasive lumbar spine surgery has dramatically evolved over the last decade. Minimally invasive techniques and transforaminal lumbar interbody fusion (TLIF) often require a steep learning curve. Surgical techniques require pre-positioning the patient in maximal kyphosis to optimize visualization of the disc space and prevent unnecessary retraction of neural structures. The authors describe their experience in validating the surgical technique recommendation of Wilson frame-induced kyphosis.

Methods. Over the past 6 months, data obtained in 20 consecutive patients (40 total levels) undergoing minimally invasive TLIF were reviewed. In each patient, preincision intraoperative radiographs were reviewed at L4-5 and L5-S1 with the patient on a Wilson frame in maximal lordosis and then in maximal kyphosis. The change in disc space angle at L4-5 and L5-S1 after changing from maximal lordosis to maximal kyphosis was reviewed. Descriptive statistics were calculated for sagittal plane angular measures at L4-5 and L5-S1 in lordosis and kyphosis, including absolute differences and percentage of change between positions. Inferential statistics were calculated using paired t-tests with $\alpha=0.05$.

Results. Twenty patients underwent single- or multilevel minimally invasive TLIF. Inducing kyphosis with the Wilson frame aided in optimizing exposure and decreasing the need for neural structure retraction. Both L4-5 and L5-S1 showed statistically significant $(\mathrm{p}<0.001)$ and clinically meaningful changes with increased segmental flexion in the kyphotic position. At L4-5 the mean increase in flexion was $4.5^{\circ}$ (95\% CI 2.9-6.0 $)$, representing an average $47 \%$ change. The mean increase in flexion at L5-S1 was $3.2^{\circ}\left(95 \%\right.$ CI $\left.2.3-4.2^{\circ}\right)$, representing an average $20.8 \%$ change. In lordosis the mean angle at L4-5 was $10.6 \pm 4.4^{\circ}$ and at L5-S1 was $17 \pm 7.0^{\circ}$. In kyphosis the mean angle at L4-5 was $6.1 \pm 4.5^{\circ}$ and at L5-S1 was $13.8 \pm 6.5^{\circ}$. Additionally, there was a statistically significant difference $(\mathrm{p}<0.05)$ in percentage of change between the 2 levels, with L4-5 showing a greater change $(27 \%$ more flexion) between positions, but the absolute mean difference between the levels was small $\left(1.3^{\circ}\right)$.

Conclusions. Minimally invasive TLIF is challenging and requires a significant learning curve. The recommended surgical technique of inducing kyphosis with the Wilson frame prior to incision significantly optimizes exposure. The authors' experience demonstrates that this technique is essential when performing minimally invasive lumbar spinal fusions. (DOI: 10.3171/2010.1.FOCUS10325)
\end{abstract}

KeY Words - Wilson frame - minimally invasive technique - lumbar fusion

$\mathrm{M}$ INIMALLY invasive spine surgery has dramatically evolved over the last decade. New techniques and instrumentation have expanded the minimally invasive field from single nerve root decompressions to multilevel instrumented spine fusions. As patients benefit from the decreased postoperative pain, blood loss, transfusion needs, and length of hospital stay, the demand for spine surgeons to become proficient in minimally invasive spine technology will only increase..$^{2-4,6,15}$

Transforaminal lumbar interbody fusion has become a procedure of choice in the management of various spine disorders requiring arthrodesis. ${ }^{12,13,17}$ Compared with the traditional posterior lumbar interbody fusion, there are clear advantages that include unilateral exposure, de-

Abbreviation used in this paper: TLIF $=$ transforaminal lumbar interbody fusion. creased neural retraction, and a lateral approach that facilitates revision surgery. However, exposure of the posterior spinal elements still requires muscle stripping and retraction that can have an adverse effect on patient outcome. ${ }^{6-11,14}$ With the advent of minimally invasive spine techniques, iatrogenic soft-tissue injury may be minimized.

Regardless of the exposure technique, pre-positioning of the patient in maximal segmental distraction while performing a TLIF is recommended. Theoretically, this optimizes visualization of the disc space and is thought to prevent unnecessary retraction of the neural structures. Once interbody spacer placement is completed, the patient is repositioned in lordosis and segmental instrumentation is secured. A common positioning device employed for this purpose is the Wilson frame.

Given the steep learning curve associated with mini- 

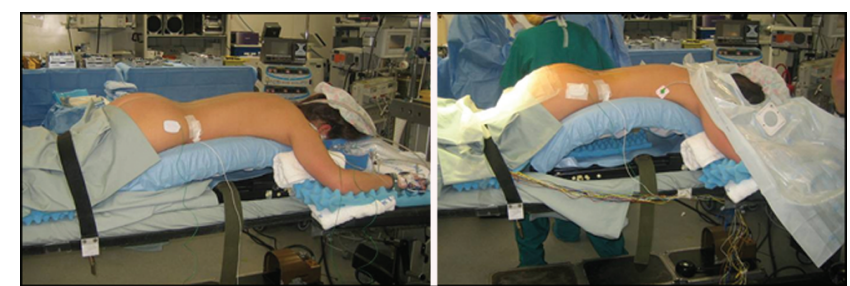
sis.

Fıg. 1. Patient positioned on the Wilson frame in lordosis and kypho-

mally invasive spinal surgery, any technique that facilitates the surgery is valued. In contrast, any procedure not necessary to the goals of the surgery becomes an unnecessary step. To this point, the literature does not quantify the disc exposure that the Wilson frame obtains in maximal lumbar kyphosis. The purpose of this study was to present our experience in validating the surgical technique recommendation of Wilson frame-induced kyphosis for interbody fixation followed by induced lordosis for final compression.

\section{Methods}

Data obtained in 20 consecutive patients (13 men, 7 women), whose average age was 44 years (range 21-61 years) and who underwent single- or multilevel minimally invasive TLIF (40 interbody levels), were retrospectively reviewed. Preoperative diagnoses included 3 cases of Meyerding Grade I isthmic spondylolisthesis (2 patients with L-4 and 1 patient with L-5 spondylolisthesis) and 17 patients with discogenic back pain due to degenerative disc disease with and without radiculopathy. No disc spaces involved in the study were collapsed by greater than $50 \%$.

All patients were preoperatively positioned on the Wilson frame (Mizuh OSI) mounted on the OSI Jackson table under the supervision of the attending surgeon. The Wilson frame was elevated to maximum position. Positioning was standardized for all patients regardless of body habitus with the abdomen allowed to hang. The hips were allowed to flex naturally over the inferior aspect of the Wilson frame onto the operating table. Knees were padded and a pillow was placed under the patient's legs at the ankle to allow the knees to flex. Arms were flexed
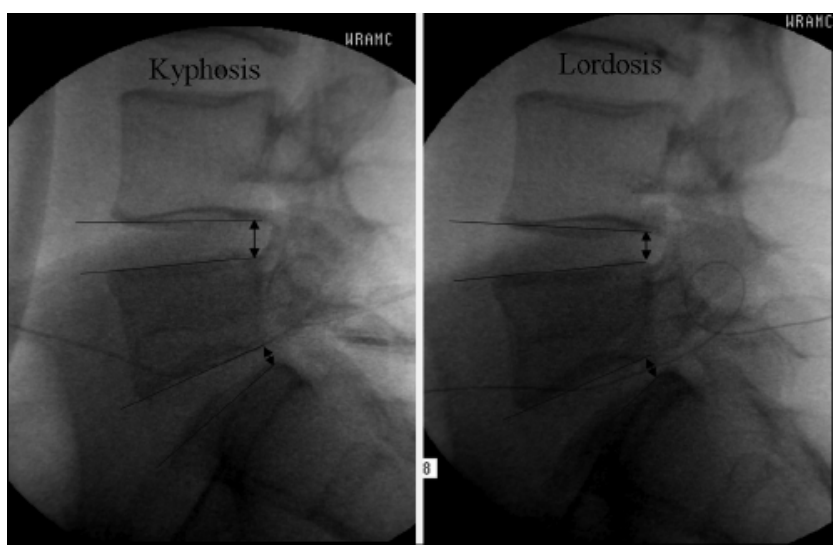

FIG. 2. Intraoperative fluoroscopic images obtained in a patient in whom kyphosis and lordosis have been induced. Arrows show intervertebral disc angles.

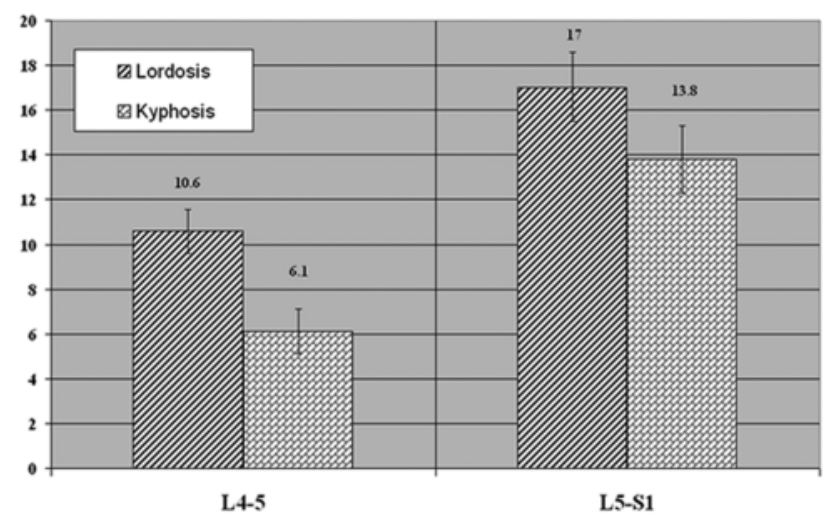

FIG. 3. Mean intervertebral angle at both $L 4-5$ and $L 5-S 1$ in both lordosis and kyphosis in 20 patients.

toward the head of the bed and supported by arm boards (Fig. 1).

Radiographs were obtained prior to preparation and draping using C-arm fluoroscopy. During confirmation of the surgical level, a radiograph was obtained with the patient in maximal kyphosis followed by maximal lordosis. The intervertebral disc angle at L4-5 (20 sides) and L5-S1 (20 sides) was analyzed using Image Pro-Plus Version 5.1 (Media Cybernetics, Inc.) (Fig. 2). Descriptive statistics were calculated for the intervertebral body angle measure at L4-5 and L5-S1 in both kyphosis and lordosis. Absolute differences and percentage of change between positions as well as inferential statistics were calculated using paired t-tests with $\alpha=0.05$ (SPSS Version 13.0). Mean values are presented \pm the SD.

\section{Results}

With the Wilson frame in the lordotic position, the mean intervertebral disc angle at L4-5 and L5-S1 was $10.6 \pm 4.4$ and $17.1 \pm 7.0^{\circ}$, respectively. With the Wilson frame inducing maximal kyphosis, the L4-5 and L5-S1 disc angles decreased to $6.1 \pm 4.5$ and $13.8 \pm 6.5^{\circ}$, respectively (Fig. 3). Radiographic analysis of the intervertebral disc angle at both L4-5 and L5-S1 showed a statistically significant change $(\mathrm{p}<0.001)$ in disc angle for both levels. In addition there was a clinically meaningful change with increased segmental flexion in the kyphotic position during the procedures. The decrease in disc angle is a result of anterior disc compression and posterior disc distraction from the adjacent vertebral body going into flexion. Consequently, the lumbar intervertebral disc angle at L4-5 and L5-S1 decreased by $4.5^{\circ}\left(95 \%\right.$ CI $\left.2.9-6.0^{\circ}\right)$ and $3.2^{\circ}$ $\left(95 \%\right.$ CI $\left.2.3-4.2^{\circ}\right)$, respectively. This represents a change of 47 and $21 \%$, respectively, at the L4-5 and L5-S1 disc levels (Table 1). As a result, there was improved posterior

TABLE 1: Mean and percentage of intervertebral disc angle change from lordosis to kyphosis

\begin{tabular}{ccc}
\hline Disc Level & $\begin{array}{c}\text { Mean Intervertebral } \\
\text { Angle Change }\left(^{\circ}\right.\end{array}$ & $\begin{array}{c}\text { \% Intervertebral } \\
\text { Angle Change }\end{array}$ \\
\hline L4-5 & 4.5 & 47 \\
L5-S1 & 3.2 & 21 \\
\hline
\end{tabular}




\section{Wilson frame and surgical exposure}

TABLE 2: Absolute and percentage of intervertebral angle change for L4-5 and L5-S1 in 20 patients

\begin{tabular}{|c|c|c|c|c|}
\hline \multirow[b]{2}{*}{ Case No. } & \multicolumn{2}{|c|}{ Absolute Change $\left({ }^{\circ}\right)$} & \multicolumn{2}{|c|}{ \% Intervertebral Angle Change } \\
\hline & $\begin{array}{l}\text { L4-5: Lordosis } \\
\text { to Kyphosis }\end{array}$ & $\begin{array}{l}\text { L5-S1: Lordosis } \\
\text { to Kyphosis }\end{array}$ & L4-5 & L5-S1 \\
\hline $1^{*}$ & 6.9 & 4.3 & 61.2 & 18.8 \\
\hline 2 & 1.0 & 1.1 & 10.0 & 17.0 \\
\hline 3 & 0.6 & -0.1 & 7.3 & -0.3 \\
\hline 4 & 1.5 & 2.0 & 28.8 & 18.6 \\
\hline 5 & 6.0 & 1.1 & 50.4 & 11.7 \\
\hline 6 & -0.4 & 4.5 & -3.0 & 35.1 \\
\hline 7 & 0.5 & 2.1 & 6.7 & 8.7 \\
\hline 8 & 2.8 & 2.9 & 18.6 & 31.0 \\
\hline 9 & 7.2 & 7.5 & 79.3 & 40.3 \\
\hline $10 \dagger$ & 8.6 & 4.4 & 199.1 & 17.0 \\
\hline 11 & 9.0 & 6.5 & 53.4 & 29.9 \\
\hline 12 & 7.5 & 2.6 & 35.4 & 10.3 \\
\hline 13 & 1.6 & 1.8 & 11.2 & 8.0 \\
\hline 14 & 2.2 & 5.0 & 31.0 & 26.5 \\
\hline 15 & 5.0 & 1.9 & 74.3 & 10.2 \\
\hline 16 & 5.5 & 4.2 & 40.6 & 15.7 \\
\hline 17 & 0.7 & 5.4 & 14.8 & 23.7 \\
\hline 18 & 9.1 & 0.5 & 63.2 & 3.5 \\
\hline 19 & 7.3 & 2.0 & 75.9 & 38.0 \\
\hline $20 \dagger$ & 6.8 & 4.2 & 81.4 & 51.4 \\
\hline
\end{tabular}

disc anulus exposure allowing for interbody instrumentation and neural element protection.

Furthermore, there was a statistically significant difference $(\mathrm{p}<0.05)$ in percentage of change between the 2 levels with L4-5 showing 27\% more flexion between positions. This is unexpected because the L5-S1 level has been reported to have a greater range of motion in flexion and extension than the L4-5 disc space. ${ }^{20}$ The increase in L4-5 flexion may be explained by lordotic positioning on the Wilson frame placing the lumbar spine in kyphosis that is mostly appreciated at the L5-S1 disc space. The absolute mean difference between L4-5 and L5-S1 was small $\left(1.3^{\circ}\right)$ and not statistically significant $(\mathrm{p}>0.05)$.

\section{Discussion}

Minimally invasive surgery has become the gold standard for many surgical specialties. ${ }^{16,21}$ The benefits of lower blood loss, shorter hospital stays, and less postoperative pain are compelling and may eventually overcome concerns of a steep learning curve..$^{2-4,15}$ Techniques that simplify as well as facilitate the surgeon's task may help to lesson any resistance associated with the technical aspects of minimally invasive spinal surgery.

Surgical techniques that involve pre-positioning of patients in maximal lumbar kyphosis optimize visualization of the disc space and minimize neural-structure retraction for intervertebral work. However, the literature supporting induced lumbar kyphosis for interbody fixa- tion is limited. Our study is the first to report disc angle changes before and after inducing lumbar kyphosis for the purpose of lumbar interbody fusion.

Placing patients in Wilson frame-induced kyphosis decreases the intervertebral disc angle and therefore maximizes posterior disc anulus exposure. By inducing lumbar kyphosis with the Wilson frame, the L4-5 and L5-S1 disc angles decreased by 47 and $21 \%$, respectively. As expected the L5-S1 intervertebral disc angle was greater than L4-5; however, the percentage of change from the lordotic to the kyphotic position on the Wilson frame was greater for the L4-5 level. The biomechanical literature has shown that flexion/extension is greatest at L5-S1. ${ }^{20}$ Therefore, it would be expected that L5-S1 would experience the greatest change in intervertebral angle. We postulate that much of this motion at L5-S1 was displaced in positioning the patient on the Wilson frame, which induces baseline kyphosis. Tan and colleagues ${ }^{19}$ noted a $50 \%$ decrease in lumbar lordosis from the standing position to being positioned on a frame that flexed the hips. Benfanti and Geissele ${ }^{1}$ reported an $8 \%$ decrease in L4-S1 segmental lordosis from the standing position to being placed on the Wilson frame. Conversely, Guanciale et al. ${ }^{5}$ noted that L5-S1 segmental lordosis was less dependent on frame type and has little change from the standing to frame position. However, it should be noted that the Wilson frame was not evaluated in the study. Nevertheless, a statistically and clinically significant decrease in lordosis was appreciated at both L4-5 and L5-S1. 
Given the small number of patients with spondylolisthesis, a correlation with intervertebral disc angle change and isthmic spondylolisthesis could not be made. One would expect an increase in intervertebral disc angle change in both settings. In both cases of L4-5 isthmic spondylolisthesis the patient had greater flexion on kyphosis (199 and 81\%) than the mean (47\%); however, in the one case of L5-S1 spondylolisthesis the patient did not have greater flexion on kyphosis (19 vs 21\%) (Table 2). In addition, partial and complete sacralized L-5 vertebrae, which have diminished motion from pseudo-transverse process articulations and rudimentary discs, would be expected to benefit only minimally from Wilson frame-induced kyphosis. In the current study there were no partial or complete sacralized L-5 vertebrae. Finally, there were no intervertebral disc levels with abutting vertebral endplates. Stokes et al. ${ }^{18}$ used biplanar radiography to evaluate lumbar coupled motion and linked advanced disc degeneration with increased coupled motion in the lower lumbar spine. Based on their work, the Wilson frame technique may increase exposure between vertebrae with a collapsed disc. To evaluate this, a follow-up study is warranted.

Minimally invasive TLIF is challenging and requires a significant learning curve. The recommended surgical technique of inducing kyphosis with the Wilson frame prior to incision significantly optimizes exposure. The Wilson frame technique may also benefit surgeons performing standard open transforaminal fusions. It is our opinion that the Wilson frame technique is essential, but only time will tell whether it becomes an accepted technique among spine surgeons.

\section{Conclusions}

Wilson frame-induced kyphosis significantly increased flexion at L4-5 and L5-S1 by 47 and 21\%, respectively. We found that the surgical technique of inducing kyphosis with the Wilson frame prior to incision significantly optimized exposure. Our experience demonstrates that this technique is essential when performing minimally invasive lumbar spinal fusions.

\section{Disclosure}

The authors report no conflict of interest concerning the materials or methods used in this study or the findings specified in this paper. The opinions and assertions contained herein are the private views of the authors and are not to be construed as official or reflecting the views of the US Army or the US Navy.

Author contributions to the study and manuscript preparation include the following. Conception and design: MK Rosner. Acquisition of data: MJ Cardoso. Analysis and interpretation of data: both authors. Drafting the article: MJ Cardoso. Critically revising the article: MJ Cardoso. Reviewed final version of the manuscript and approved it for submission: MK Rosner. Statistical analysis: MJ Cardoso. Administrative/technical/material support: both authors. Study supervision: MK Rosner.

\section{References}

1. Benfanti PL, Geissele AE: The effect of intraoperative hip position on maintenance of lumbar lordosis: a radiographic study of anesthetized patients and unanesthetized volunteers on the Wilson frame. Spine 22:2299-2303, 1997

2. Foley KT, Holly LT, Schwender JD: Minimally invasive lumbar fusion. Spine 28 (15 Suppl):S26-S35, 2003
3. Foley KT, Lefkowitz MA: Advances in minimally invasive spine surgery. Clin Neurosurg 49:499-517, 2002

4. German JW, Foley KT: Minimal access surgical techniques in the management of the painful lumbar motion segment. Spine 30 (16 Suppl):S52-S59, 2005

5. Guanciale AF, Dinsay JM, Watkins RG: Lumbar lordosis in spinal fusion. A comparison of intraoperative results of patient positioning on two different operative table frame types. Spine 21:964-969, 1996

6. Holly LT, Schwender JD, Rouben DP, Foley KT: Minimally invasive transforaminal lumbar interbody fusion: indications, technique, and complications. Neurosurg Focus 20(3):E6, 2006

7. Kawaguchi $\mathrm{Y}$, Matsui $\mathrm{H}$, Tsuji $\mathrm{H}$ : Back muscle injury after posterior lumbar spine surgery. A histologic and enzymatic analysis. Spine 21:941-944, 1996

8. Kawaguchi Y, Matsui H, Tsuji H: Back muscle injury after posterior lumbar spine surgery. Part 1: Histologic and histochemical analyses in rats. Spine 19:2590-2597, 1994

9. Kawaguchi Y, Matsui H, Tsuji H: Back muscle injury after posterior lumbar spine surgery. Part 2: Histologic and histochemical analyses in humans. Spine 19:2598-2602, 1994

10. Kawaguchi Y, Yabuki S, Styf J, Olmarker K, Rydevik B, Matsui $\mathrm{H}$, et al: Back muscle injury after posterior lumbar spine surgery. Topographic evaluation of intramuscular pressure and blood flow in the porcine back muscle during surgery. Spine 21:2683-2688, 1996

11. Kotil K, Tunckale T, Tatar Z, Koldas M, Kural A, Bilge T: Serum creatine phosphokinase activity and histological changes in the multifidus muscle: a prospective randomized controlled comparative study of discectomy with or without retraction. J Neurosurg Spine 6:121-125, 2007

12. Lowe TG, Tahernia AD, O'Brien MF, Smith DA: Unilateral transforaminal posterior lumbar interbody fusion (TLIF): indications, technique, and 2-year results. J Spinal Disord Tech 15:31-38, 2002

13. Moskowitz A: Transforaminal lumbar interbody fusion. Orthop Clin North Am 33:359-366, 2002

14. Ozgur BM, Hughes SA, Baird LC, Taylor WR: Minimally disruptive decompression and transforaminal lumbar interbody fusion. Spine J 6:27-33, 2006

15. Park Y, Ha JW: Comparison of one-level posterior lumbar interbody fusion performed with a minimally invasive approach or a traditional open approach. Spine 32:537-543, 2007

16. Porte RJ, De Vries BC: Laparoscopic versus open cholecystectomy: a prospective matched-cohort study. HPB Surg 9:71-75, 1996

17. Rosenberg WS, Mummaneni PV: Transforaminal lumbar interbody fusion: technique, complications, and early results. Neurosurgery 48:569-575, 2001

18. Stokes IA, Wilder DG, Frymoyer JW, Pope MH: 1980 Volvo award in clinical sciences. Assessment of patients with lowback pain by biplanar radiographic measurement of intervertebral motion. Spine 6:233-240, 1981

19. Tan SB, Kozak JA, Dickson JH, Nalty TJ: Effect of operative position on sagittal alignment of the lumbar spine. Spine 19:314-318, 1994

20. White AA: Clinical Biomechanics of the Spine, ed 2. Philadelphia: Lippincott Williams \& Wilkins, 1990

21. Zacks SL, Sandler RS, Rutledge R, Brown RS Jr: A populationbased cohort study comparing laparoscopic cholecystectomy and open cholecystectomy. Am J Gastroenterol 97:334-340, 2002

Manuscript submitted December 23, 2009.

Accepted January 28, 2010.

Address correspondence to: Mario J. Cardoso, M.D., Neurosurgery Service, Walter Reed Army Medical Center, 6900 Georgia Avenue NW, Washington, DC 20307. email: mtcardoso@comcast. net. 